\title{
Fatores associados a enteroparasitoses em escolares da rede municipal de ensino de Cambé
}

\section{Factors associated with intestinal parasites in schoolchildren of municipal schools in Cambé}

\author{
Fabiana Maria Ruiz Lopes-Mori ${ }^{1}$, Regina Mitsuka-Breganó ${ }^{2}$, Francisco José de \\ Abreu Oliveira ${ }^{3}$, Maria Cláudia de Menezes Noronha Dutra ${ }^{4}$, Maria de Brito Lô \\ Sarzi $^{5}$, Marta Radigonda Aidar ${ }^{6}$, Ivete Conchon-Costa ${ }^{7}$
}

\section{Resumo}

O objetivo deste trabalho foi determinar os fatores associados à prevalência das parasitoses intestinais em escolares da rede municipal de Cambé, Paraná. Foram analisadas 1996 amostras de fezes, coletadas entre 2006 e 2009, pelos métodos de Hoffmann, Pons e Janer (1934), de Faust e colaboradores e de Kato-Katz. A prevalência encontrada foi de 23,2\%. Os parasitas encontrados foram Entamoeba coli (10,4\%); Endolimax nana (9,6\%), Giardia lamblia (6,4\%), Enterobius vermicularis $(1,5 \%)$, Entamoeba histolytica/dispar (0,3\%), Trichuris trichiura (0,4\%), Iodamoeba butschlii, Hymenolepis nana e Ancilostomídeos (0,2\%) e Ascaris lumbricoides e Schistosoma mansoni (0,1\%). Não houve diferença estatisticamente significativa com relação ao sexo. A faixa etária, a renda familiar, o grau de instrução da mãe, o consumo de água não tratada, a ausência de esgoto e o hábito da criança frequentar córregos apresentaram associação com a presença de enteroparasitas. Apesar da maior prevalência ser de protozoários comensais este dado é preocupante, pois indica que a transmissão fecal-oral está presente nesta população podendo ocorrer aumento na transmissão das formas patogênicas, já que estas possuem as mesmas vias de transmissão. Foram identificados casos de esquistossomose que não eram autóctones, porém o diagnóstico precoce desta infecção foi importante para se evitar a contaminação do meio ambiente.

Palavras chave: Doenças parasitárias. Pré-escolar. Fatores de risco.

\begin{abstract}
The aim of this work was to determine factors associated with the prevalence of intestinal parasites in schoolchildren in the municipality of Cambé, Paraná. A total of 1996 stool samples were collected between 2006 and 2009, using the methods of Hoffman, Pons and Janer, Faust and collaborators and the Kato-Katz. The prevalence was $23.2 \%$. The parasites found were Entamoeba coli (10.4\%); Endolimax
\end{abstract}

1 Docente de Parasitologia, Departamento de Biomedicina, Centro Universitário Filadélfia, UniFil, Londrina, Paraná, Brasil. E-mail: fabiana.mori@unifil.br

${ }^{2}$ Docente de Parasitologia, Departamento de Ciências Patológicas, Centro de Ciências Patológicas, Universidade Estadual de Londrina (UEL), Paraná, Brasil. E-mail: rbregano@gmail.com.

${ }^{3}$ Docente de Parasitologia, Departamento de Ciências Patológicas, Centro de Ciências Patológicas, Universidade Estadual de Londrina (UEL), Paraná, Brasil. E-mail: abreu@uel.br.

${ }^{4}$ Docente de Parasitologia, Departamento de Ciências Patológicas, Centro de Ciências Patológicas, Universidade Estadual de Londrina (UEL), Paraná, Brasil. E-mail: menezes@uel.br.

${ }^{5}$ Secretaria Municipal de Saúde de Cambe, Paraná, Brasil. E-mail: mariabrito@hotmail.com.

${ }^{6}$ Secretaria Municipal de Educação de Cambe, Paraná, Brasil. E-mail: martaaidar@hotmail.com.

${ }^{7}$ Docente de Parasitologia, Departamento de Ciências Patológicas, Centro de Ciências Patológicas, Universidade Estadual de Londrina (UEL), Paraná, Brasil. E-mail: conchon@uel.br. 
nana (9.6\%), Giardia lamblia (6.4\%), Enterobius vermicularis (1.5\%), Entamoeba histolytica/dispar $(0.3 \%)$, Trichuris trichiura (0.4\%), Iodamoeba butschlii, Hymenolepis nana and hookworm infection $(0.2 \%)$ and Ascaris lumbricoides and Schistosoma mansoni (0.1\%). We found no statistically significant gender differences. The age, family income, mother's education level, consumption of untreated water, absence of sewage collection and contact with freshwater streams were associated with the presence of intestinal parasites. Although the highest prevalence of protozoa is commensal this is worrying as it indicates that the fecal-oral transmission is present in this population and may increase the transmission of pathogenic forms, since they share the same transmission routes. The identified cases of schistosomiasis were not autochthonous, but early diagnosis of this infection was important to avoid contamination of the environment.

Keywords: Parasitic diseases. Preschool. Risk factors.

\section{Introdução}

As parasitoses intestinais representam um grave problema de saúde pública mundial, e, geralmente, estão associadas a algumas características da população como o baixo nível socioeconômico (BISCEGLI et al., 2009), o clima, as más condições sanitárias e a falta de informações (LOPES et al., 2006). Embora haja uma vasta literatura sobre a importância destas infecções, pouca atenção tem sido dada ao assunto (FERREIRA; ANDRADE, 2005), além disso, faltam dados disponíveis sobre morbidade e mortalidade decorrentes das parasitoses intestinais que, geralmente, não são notificadas pelos serviços de saúde (BISCEGLI et al., 2009).

Os efeitos patogênicos causados pelas infecções parasitárias exercem importante influência sobre o estado nutricional, o crescimento e a função cognitiva, principalmente nas fases precoces da vida (FERREIRA et al., 2004) ocasionando quadros de má-absorção, diarreia crônica, anemia, desnutrição, dores abdominais, dificuldade de aprendizado e concentração, atraso no crescimento e baixo rendimento escolar (CASTRO et al., 2005; PITTNER et al., 2007).

Em um estudo multicêntrico realizado em escolares de 7 a 14 anos em 10 estados brasileiros, $55,3 \%$ dos estudantes foram diagnosticados com algum tipo de enteroparasitose sendo que a ascaridiose, a tricurose e a giardíase apresentaram distribuição regular (CAMPOS et al., 1988), portanto, ações sanitárias e educativas, associadas ao tratamento, são necessárias para um controle efetivo dessas enfermidades (LOPES et al., 2006).

No estado do Paraná, Lopes et. al (2006) encontraram uma prevalência de $68,2 \%$ de enteroparasitas em crianças em idade escolar da cidade de Jataizinho. Pittner et al. (2007) encontraram $60,5 \%$ na cidade de Guarapuava. Estes achados reforçam a importância da pesquisa destes parasitas na população a fim de implantar medidas de controle específicas e melhorar a qualidade de vida das pessoas.

O objetivo deste estudo foi determinar os fatores associados à infecção por parasitas intestinais em escolares da rede municipal de Cambé, Paraná.

\section{Material e Métodos}

O município de Cambé está localizado no norte do estado do Paraná, possui 22 escolas municipais com, aproximadamente 5555 alunos matriculados nos ensino pré-escolar e fundamental (CAMBÉ, 2010). Participaram deste estudo 1996 alunos com idade de cinco a 15 anos. A amostragem utilizada neste estudo foi superior ao estimado estatisticamente para uma prevalência de 50\%, com intervalo de confiança de 95\% (DEAN et al., 1994), que foi de 718 amostras.

Após o consentimento pós-informado do responsável pela criança, procedeu-se a aplicação de um questionário com informações sobre os hábitos alimentares, higiênicos e socioeconômicos. Estes dados foram analisados pelo programa 
EpiInfo ${ }^{5}$, utilizando-se o teste de qui-quadrado ou exato de Fisher, quando adequado, para verificar a significância estatística e o Odds Ratio (OR) para avaliar a associação entre as variáveis pesquisadas e a presença de enteroparasitas, com nível de significância de 5\%.

Foi coletada uma amostra de material fecal de cada criança, entre 2006 e 2009, que foram submetidas aos exames coproparasitológicos no laboratório de Parasitologia da Universidade Estadual de Londrina (UEL). As crianças que apresentaram resultados positivos foram encaminhadas às Unidades Básicas de Saúde do município para o tratamento.

O método de Faust e et al. (1939) é uma técnica baseada na centrífugo-flutuação em sulfato de zinco onde $10 \mathrm{~g}$ de fezes são diluídos em $20 \mathrm{ml}$ de água filtrada, que deverá ser homogeneizada e filtrada, através de uma gaze dobrada em quatro. O filtrado é transferido para um tubo de ensaio e centrifugado a $2500 \mathrm{rpm}$, por um minuto. O líquido sobrenadante é desprezado e o sedimento ressuspendido em água. A centrifugação e a ressuspensão deve ser repetida mais três ou quatro vezes até que o líquido fique claro. Após a última lavagem o sedimento é ressuspendido com uma solução de sulfato de zinco a $33 \%$, densidade de $1,18 \mathrm{~g} / \mathrm{ml}$ que deverá ser centrifugado novamente a $2500 \mathrm{rpm}$ por um minuto. Os cistos e ovos leves presentes estarão na película superficial, que será recolhida com alça de platina, colocada numa lâmina, junto a uma gota de lugol, e coberta com lamínula.

O método de Hoffman, Pons e Janer (1934) ou método de sedimentação espontânea, utiliza aproximadamente, $2 \mathrm{~g}$ de fezes que deve ser colocada em um frasco próprio, com cerca de 5 ml de água e homogeneizados com um bastão de vidro. Deve-se serão acrescentar mais $20 \mathrm{ml}$ de água e a filtrar a solução para um cálice cônico de $200 \mathrm{ml}$ de capacidade, utilizando uma gaze dobrada em quatro. O volume de água no cálice é completado e a suspensão deverá ficar em repouso por até 24 horas. Após este tempo, parte do sedimento deverá ser colocado numa lâmina, com auxílio de uma pipeta, acrescido de uma gota de lugol, coberto com lamínula e examinado ao microscópio óptico. Deverá ser analisado, no mínimo, duas lâminas de cada amostra.

A técnica descrita por Kato e modificada por Katz e Peixoto (2000), consiste em colocar a amostra a ser analisada sobre um pedaço de papel higiênico e comprimir com um pedaço de tela metálica similar a náilon. Com o auxílio de um palito, as fezes que passarem para a parte superior da tela e transferidas para o orifício $(6 \mathrm{~mm}$ de diâmetro) de um cartão retangular de plástico, é colocado sobre uma lâmina de microscopia. Após encher completamente o orifício, o cartão será retirado cuidadosamente, deixando as fezes sobre a lâmina de vidro (aproximadamente 42g). A amostra deverá ser coberta com lamínula de papel celofane e a lâmina deverá ser invertida sobre uma folha de papel absorvente e comprimida. A contagem dos ovos deverá ser feita de uma a duas horas após a preparação da lâmina e a leitura será feita em microscópio óptico. O número de ovos encontrados, multiplicado por 23 , corresponderá ao número de ovos por grama de fezes.

A pesquisa foi aprovada pelo Comitê de Ética em Pesquisa da UEL (parecer 165/07).

\section{Resultados}

Das 1996 crianças pesquisadas, 86,9\% eram provenientes da zona urbana e $13,1 \%$ da zona rural. Quanto ao sexo, 48,6\% (971/1996) eram escolares do sexo masculino e 51,4\% (1025/1996) do sexo feminino.

A prevalência de parasitos intestinais foi de 23,2\% (464/1996). Entre as espécies encontradas, 20,8\% (415/1996) foram protozoários, 1,7\% (35/1996) helmintos e $0,7 \%$ (14/1996) protozoários e helmintos. A maioria das crianças apresentou apenas uma única espécie de parasita (18,3\%, 366/1996). Não houve diferença estatisticamente significativa com relação ao sexo $(p=0,85)$, pois, 
entre as 971 amostras de meninos, 228 (23,5\%) estavam parasitadas e das 1025 amostras de meninas, $236(23,0 \%)$ estavam parasitadas. A faixa etária mais acometida foi de 7 a 10 anos $(53,1 \%)$.

Entre os protozoários, a espécie mais frequente foi a Entamoeba coli (10,4\%, 208/1996); seguida do Endolimax nana (9,6\%, 192/1996), Giardia lamblia (6,4\%, 128/1996), Entamoeba histolytica/dispar (0,3\%, 6/1996) e Iodamoeba butschlii (0,2\%, 4/1996). Nas amostras positivas para helmintos, a espécie mais prevalente foi o Enterobius vermicularis $(1,5 \%$, 29/1996), seguido do Trichuris trichiura (0,4\%, 8/1996), Hymenolepis nana e Ancilostomídeos
$(0,2 \%, 4 / 1996)$ e Ascaris lumbricoides e Schistosoma mansoni (0,1\%, 2/1996).

Dados de saneamento da população estudada mostraram que 4,3\% das crianças não utilizam água tratada, $0,5 \%$ não utilizam a rede pública como destino para o esgoto e $11,5 \%$ não utilizam privada. Além disso, $10,2 \%$ das crianças frequentam córregos para atividades de lazer.

As variáveis faixa etária, renda familiar, grau de instrução da mãe, o consumo de água não tratada, ausência de rede de esgoto e o hábito da criança frequentar córregos apresentaram associação com a presença de enteroparasitas (Tabela 1).

Tabela 1 - Análise das variáveis associadas à presença de enteroparasitas em escolares da rede municipal de ensino de Cambé, Paraná, 2006-2009.

\begin{tabular}{|c|c|c|c|}
\hline Variável & $\begin{array}{c}\text { Amostras positivas/ } \\
\text { total }(\%)\end{array}$ & $\mathbf{P}$ & OR $^{*}($ IC $95 \%)$ \\
\hline \multicolumn{4}{|c|}{ Residência } \\
\hline Zona urbana & $392 / 1710(22,9)$ & 0,4479 & $0,87(0,64-1,18)$ \\
\hline \multirow[t]{2}{*}{ Zona rural } & $65 / 257(25,3)$ & & \\
\hline & Sexo & & \\
\hline Masculino & $228 / 971(23,4)$ & 0,8506 & $1,02(0,83-1,25)$ \\
\hline \multirow[t]{2}{*}{ Feminino } & $236 / 1025(23,20)$ & & \\
\hline & Faixa etária & & \\
\hline$>7$ anos & $327 / 1214(26,9)$ & $<0,001$ & $1,73(1,37-2,18)$ \\
\hline \multirow[t]{2}{*}{ Até 6 anos } & $137 / 780(17,5)$ & & \\
\hline & \multicolumn{2}{|c|}{ Grau de instrução da mãe } & \\
\hline Até 8 anos de estudo & $325 / 1253(25,9)$ & $<0,001$ & $1,77(1,38-2,28)$ \\
\hline \multirow[t]{2}{*}{ Mais de 8 anos de estudo } & $107 / 649(16,4)$ & & \\
\hline & Renda familia & & \\
\hline Até 2 salários mínimos & $312 / 1220(25,5)$ & $<0,001$ & $1,61(1,27-2,05)$ \\
\hline \multirow[t]{2}{*}{ Mais de 2 salários mínimos } & $121 / 688(17,5)$ & & \\
\hline & Consumo de água tı & & \\
\hline Não & $33 / 85(38,8)$ & $<0,001$ & $2,18(1,36-3,50)$ \\
\hline Sim & $430 / 1909(22,5)$ & & \\
\hline
\end{tabular}




\begin{tabular}{|c|c|c|c|}
\hline Variável & $\begin{array}{c}\text { Amostras positivas/ } \\
\text { total }(\%)\end{array}$ & $\mathbf{P}$ & OR* (IC 95\%) \\
\hline \multicolumn{4}{|c|}{ Presença de esgoto } \\
\hline Não & $352 / 1411(24,9)$ & $<0,001$ & $1,43(1,12-1,84)$ \\
\hline Sim & $109 / 579(18,8)$ & & \\
\hline \multicolumn{4}{|c|}{ Utiliza privada } \\
\hline Sim & $400 / 1733(23,0)$ & 0,9404 & $0,97(0,70-1,35)$ \\
\hline Não & $53 / 225(23,6)$ & & \\
\hline \multicolumn{4}{|c|}{ Coleta de lixo } \\
\hline Sim & $410 / 1793(22,9)$ & 0,2885 & $0,81(0,58-1,15)$ \\
\hline Não & $50 / 188(26,6)$ & & \\
\hline \multicolumn{4}{|c|}{ Casa de alvenaria } \\
\hline Sim & $392 / 1712(22,9)$ & 0,2850 & $0,84(0,62-1,15)$ \\
\hline Não & $69 / 295(23,3)$ & & \\
\hline \multicolumn{4}{|c|}{ Frequenta córrego } \\
\hline Sim & $63 / 203(31,0)$ & $<0,001$ & $1,59(1,14-2,15)$ \\
\hline Não & $357 / 1780(20,0)$ & & \\
\hline \multicolumn{4}{|c|}{ Presença de horta em casa } \\
\hline Sim & $101 / 401(25,1)$ & 0,3316 & $1,14(0,88-1,47)$ \\
\hline Não & $345 / 1518(22,7)$ & & \\
\hline \multicolumn{4}{|c|}{ Presença de animal doméstico } \\
\hline Sim & $353 / 1484(23,7)$ & 0,4547 & $1,10(0,86-1,41)$ \\
\hline Não & $106 / 482(22,0)$ & & \\
\hline
\end{tabular}

* OR: Odds Ratio

Fonte: Autor.

\section{Discussão}

A prevalência de parasitas intestinais encontrada neste estudo $(23,3 \%)$ foi baixa quando comparada a outras pesquisas realizadas no estado do Paraná, como por exemplo, em Jataizinho $(68,2 \%)$ e em Guarapuava $(60,5 \%)$ e em outros estados como
Rio Grande do Sul (70,5\%), Bahia $(66,1 \%)$ e em Goiás (39,9\%) (LOPES et al., 2006; PITTNER et al., 2007; PRADO et al., 2001; QUADROS et al., 2004; ZAIDEN et al., 2008). No presente estudo a prevalência encontrada pode estar associada aos dados de saneamento da população onde apenas $4,3 \%$ das crianças não utilizam água tratada, $0,5 \%$ 
não utilizam a rede pública como destino para o esgoto e $11,5 \%$ não utilizam privadas.

Analisando-se a frequência de mono e poliparasitismo observou-se que 78,9\% das crianças estavam parasitadas por apenas uma espécie de parasito, e 22,1\% das crianças estavam parasitadas por mais de uma espécie de parasito. Pittnner et al. (2007) também encontraram uma maior frequência de monoparasitismo em escolares de Guarapuava, Paraná (76,4\%), assim como Prado et al. (2001) em escolares de Salvador, Bahia (66,1\%). Segundo Rocha, Braz e Calheiros (2010) as precárias condições de saneamento básico expõem as populações à aquisição de diferentes enteroparasitos, tornando frequentes os casos de poliparasitismo, situação não vivida por esta população estudada; conforme citado acima. Contudo, muitas regiões no Brasil como em Bambuí, Minas Gerais, os escolares apresentaram maior prevalência de poliparasitismo (79,9\%) (ROCHA et al., 2000), bem como em Jataizinho, Paraná (50,5\%) (LOPES et al., 2006).

Observamos ainda neste estudo que a frequência das enteroparasitoses foi inversamente proporcional à faixa etária do hospedeiro. A partir da análise das variáveis observou-se que crianças acima dos sete anos de idade tem 1,73 mais chances de adquirir enteroparasitoses. Segundo Santos e Merlini (2010) crianças até nove anos de idade tem atividades de lazer geralmente em ambientes externos, podendo ter contatos mais frequentes com geohelmintos, enquanto crianças mais velhas estão mais restritas a ambientes fechados.

Os protozoários encontrados mais frequentes foram a Entamoeba coli $(10,5 \%)$ e a Endolimax nana (9,8\%), assim como os achados de Lopes et al. (2006) em escolares de Jataizinho, Paraná. Apesar de serem considerados comensais, a presença destes parasitos é alvo de grande preocupação por possuírem o mesmo mecanismo de transmissão de outros enteroparasitas (via fecal-oral) e, portanto, servem de bom indicador das condições higiênicosanitárias da população.
O parasito patogênico mais prevalente foi a Giardia lamblia (6,3\%) e, segundo Vidal e Catapani (2005), no Brasil a prevalência de giardíase varia de $9 \%$ a $50 \%$, especialmente em crianças com mais de quatro anos de idade. Apesar da baixa frequência, a ocorrência deste parasito provavelmente é devida à falta de hábitos higiênicos-sanitários nos casos positivos já que o consumo de água não tratada e a ausência de esgoto apresentaram associação com a presença de enteroparasitas na população estudada. Resultados semelhantes foram encontrados por Rocha et al. (2000) em Bambuí, Minas Gerais, onde as crianças apresentaram $6,2 \%$ de positividade para este protozoário.

Machado et al. (1999) sugerem que os níveis socioeconômicos e culturais influenciam diretamente as condições de higiene pessoal e cuidados com a água e os alimentos, podendo, deste modo, inferir que em classes menos favorecidas estes cuidados não são rigorosamente observados. Além disso, Biscegli et al. (2009) relatam que a elevada frequência deste parasito pode estar associada ao hábito de ingerir água não filtrada e/ou não fervida e ao fato de os cistos serem resistentes ao tratamento da água.

Foi observada uma baixa frequência de helmintos sendo que a maior frequência foi de $E$. vermicularis $(1,6 \%)$ assemelhando-se aos resultados de Zochio et al. (2006) em escolares de Bauru, São Paulo, mas difere de outros trabalhos onde a presença de $A$. lumbricoides aparece com maior frequência entre crianças em idade escolar (LOPES et al., 2006; PITTNER et al., 2007; QUADROS et al., 2004; ROCHA et al., 2000; ZAIDEN et al., 2008).

A presença de ancilostomídeos em $0,2 \%$ das amostras apresenta resultados inferiores aos encontrados por Lopes et al. (2006), em escolares de Jataizinho, Paraná, e por Chieffi et al. (1988) (1,3\%) em escolares de Guarulhos, São Paulo, e, segundo Lopes et al. (2006), este fato pode estar associado a alguns fatores como a urbanização, menos contato com a terra e melhoria das condições de 
saneamento nas últimas décadas. Sabe-se ainda, que a disseminação das helmintíases é dependente das condições de umidade do solo, já que os seus ovos, quando liberados no solo pelas fezes do hospedeiro definitivo, não possuem capacidade de infecção, mas podem contaminar água e alimentos (GROSS et al., 1989), e, apesar de Cambé apresentar clima subtropical úmido mesotérmico, possui verões quentes e invernos com geadas (CAMBÉ, 2010), fato este que pode prejudicar a proliferação destes parasitos. Ainda segundo Biscegli et al. (2009), a baixa frequência de helmintíases, pode estar relacionada ao uso sistemático de automedicação, tão comum em nosso meio.

Quanto ao Schistosoma mansoni, duas amostras $(0,1 \%)$ foram positivas, ambas residentes na zona urbana. Estes casos foram prontamente relatados à Vigilância Epidemiológica do município, que realizou uma busca ativa e detectou serem casos não autóctones. Assim, o diagnóstico precoce desta infecção foi importante para se evitar a contaminação do meio ambiente e disseminação da infecção.

A renda familiar baixa apresentou significância estatística com a presença de enteroparasitas, onde crianças cuja família recebe até dois salários mínimos tem 1,61 mais chances de adquirir enteroparasitoses $(p<0,001)$, e, crianças cuja mãe possui até oito anos de estudo há 1,77 mais chance de adquirir parasitoses intestinais $(p<0,001)$. Resultados semelhantes foram observados por Machado et al. (1999) que encontraram percentuais mais elevados de giardíase e de helmintíases nas crianças cujos pais possuíam baixa escolaridade. Os autores sugerem que os níveis socioeconômico e cultural influenciam as condições de higiene pessoal e cuidados com a água e os alimentos, podendose inferir que em classes menos favorecidas estes cuidados não são rigorosamente observados.

O consumo de água não tratada $(\mathrm{p}<0,001)$ e a ausência de rede de esgoto $(p=0,0039)$ também apresentaram associação com a presença de enteroparasitas. Este dado é preocupante já que a elevada frequência de protozoários $(20,8 \%$, 415/1996) encontrada reflete hábitos higiênicossanitários precários da população. $\mathrm{O}$ hábito da criança frequentar córregos $(p<0,001)$ também apresentou associação com a presença de enteroparasitas. Resultados semelhantes foram encontrados por Lopes et al. (2006) em escolares de Jataizinho, Paraná, onde o principal fator associado as parasitoses intestinais foi o hábito de frequentar rio, riacho ou lagoa $(\mathrm{OR}=2,78$; IC $95 \% ; 1,32-5,92)$.

Outras variáveis foram analisadas quanto à associação com a presença de enteroparasitas, mas não apresentaram significância estatística. Segundo Machado et al. (1999) a influência de outros fatores na determinação das parasitoses intestinais, como por exemplo, o sexo, a residência (zona urbana e rural) e o contato com animais domésticos não está totalmente estabelecida.

\section{Conclusões}

Sabe-se que a presença de protozoários comensais é preocupante, pois indica que há disseminação destas formas nos alimentos e na água, favorecendo a transmissão destes organismos não patogênicos, podendo ocasionar um aumento na transmissão das formas patogênicas, uma vez que ambas apresentam as mesmas vias de transmissão.

O estudo da ocorrência de enteroparasitoses em pré-escolares é um dos indicadores utilizados para avaliar as condições higiênico-sanitárias da comunidade, fato este que pode subsidiar ações de controle e programas de educação sanitária, com o tratamento das crianças parasitadas e mudanças nas condições ambientais nestas populações, para que haja melhoria de vida dos habitantes dessas comunidades.

\section{Agradecimentos}

À Secretaria Municipal de Saúde do município de Cambé e a Secretaria Municipal de Educação do município de Cambé. 


\section{Referências}

BISCEGLI, T. S.; ROMERA, J.; CANDIDO, A. B.; SANTOS, J. M.; CANDIDO, E. C. A.; BINOTTO, A. L. Estado nutricionale prevalência de enteroparasitoses em crianças matriculadas em creche. Revista Paulista de Pediatria, São Paulo, v. 27, n. 3, p. 289-295, 2009.

CAMBÉ. Prefeitura Municipal. Dados gerais. Disponível em: <http:/www.cambe.pr.gov.br/site/ cambe/dadosgeraiscambe.html.>. Acesso em: 8 jan. 2010 .

CAMPOS, R.; BRIQUES, W.; BELDA NETO, M.; SOUZA, J. M.; KATZ, N.; SALATA, E.; DACAL, A. R. G.; DOURADO, H.; CASTANHO, R. E. P.; GURVITZ, R.; ZINGANO, A.; PEREIRA, G. J. M.; FERRIOLLI FILHO, E.; CAMILO-COURA, L.; FARIA, J. A. S.; CIMERMAM, B.; SIQUEIRA FILHO, J. B.; PRATA, A. Levantamento multicêntrico de parasitoses intestinais no Brasil. São Paulo: Rhodia, 1988.

CASTRO, T. G.; NOVAES, J. F.; SILVA, M. R.; COSTA, N. M. B.; FRANCESCHINI, S. C. C.; TINOCO, A. L. A.; LEAL, P. F. G. Caracterização do consumo alimentar, ambiente socioeconômico e estado nutricional de pré-escolares de creches municipais. Revista de Nutrição, Campinas, v. 18, n. 3, p. 321-330, 2005.

CHIEFFI, P. P.; WALDMAN, E. A.; SOUZA DIAS, R. M. D.; TORRES, D. M. A. G. V.; CHIMARA, R.; MIZUMOTO, L. C.; DA SILVA, A. M. A.; UEHARA, M. Enteroparasitoses no município de Guarulhos, SP, Brasil. Prevalência de infecção entre escolares residentes no bairro de Taboão, em junho de 1984. Revista do Instituto Adolfo Lutz, São Paulo, v. 48, p. 75-80, 1988.

DEAN, A. G.; DEAN, J. A.; COULOMERIER, D.; BRENDEL, K. A.; SMITH, D. C.; BURTON, A. H.; DICKER, R. C.; SULIVAN, K. M.; FAGAN, R. F.; ARNER, T. G. Epi info, version 6: a word processing, data bases, and statistic program for epidemiology on microcomputers. Atlanta: Center for Diseases Control and Prevention, 1994.

FAUST, E. C.; SAWITZ, W.; TOBIE, J.; ODOM, V.; PERES, C.; LINEICOME, D. E. Comparative efficiency of various technics for the dignosis of protozoa and helminths in feces. The Journal of Parasitology, Lawrence, v. 25, n. 3, p. 241-262, 1939.
FERREIRA, G. R.; ANDRADE, C. F. S. Alguns aspectos socioeconômicos relacionados a parasitoses intestinais e avaliação de uma intervenção educativa em escolares de Estiva Gerbi, SP. Revista do Instituto de Medicina Tropical de São Paulo, São Paulo, v. 38, n. 5, p. 402-405, 2005.

FERREIRA, J. R.; VOLPATO, F.; CARRICONDO, F. M.; MARTINICHEN, J. C.; LENARTOVICZ, V. Diagnóstico e prevenção de parasitoses no reassentamento São Francisco em Cascavel - PR. Revista Brasileira de Análises Clínicas, Rio de Janeiro, v. 36, p. 145-146, 2004.

GROSS, R.; SCHELL, B.; MOLINA, M. C. B.; LEÃO, M. A. C.; STRACK, U. The impact of improvement of water supply and sanitation facilities on diarrhea and intestinal parasites: a Brazilian experience with children in two low-income urban communities. Revista de Saúde Pública, São Paulo, v. 23, n. 3, p. 214-220, 1989.

HOFFMANN, W. A.; PONS, J. A.; JANER, J. L. The sedimentation concentration method in schistosomiasis mansoni. Journal of Public Health and Tropical Medicine, Oxford, v. 9, p. 283-291, 1934.

KATZ, N.; PEIXOTO, S. N. Análise crítica da estimativa do número de portadores de esquistossomose no Brasil. Revista da Sociedade Brasileira de Medicina Tropical, Uberaba, v. 33, n. 3, p. 303-308, 2000.

LOPES, F. M. R.; GONÇALVES, D. D.; REIS, C. R.; MITSUKA-BREGANÓ, R.; ANARUMA FILHO, F.; MURAD. V. A.; MENEZES, M. C. N. D.; FREIRE, R. L.; FREITAS, J. C.; SANTANA, M. A. Z.; NAVARRO, I. T. Occurrence of enteroparasitosis in schoolchildren of the municipal district of Jataizinho, State of Paraná, Brazil. Acta Scient and Health Sciencie, Maringá, v. 28, n. 2, p. 107-111, 2006.

MACHADO, R. C.; MARCARI, E. L.; CRISTANTE, S. F. V.; CARARETO, C. M. A. Giardíase e helmintíases em crianças de creches e escolas de $1^{\circ}$ e $2^{\circ}$ graus (públicas e privadas) da cidade de Mirassol (SP, Brasil). Revista da Sociedade Brasileira de Medicina Tropical, Uberaba, v. 32, v. 6, p. 697-704, 1999. 
PITTNER, E.; MORAES, I. F.; SANCHES, H. F.; TRINCAUS, M. R.; RAIMONDO, M. L.; MONTEIRO, M. C. Enteroparasitoses em crianças de uma comunidade escolar na cidade de Guarapuava, PR. Revista Salus, Guarapuava, v. 1, p. 97-100, 2007.

PRADO, M. S.; BARRETO, M. L.; STRINA, A.; FARIA, J. A. S.; NOBRE, A. A.; JESUS, S. R. Prevalência e intensidade da infecção por parasitas intestinais em crianças na idade escolar na Cidade de Salvador (Bahia, Brasil). Revista da Sociedade Brasileira de Medicina Tropical, Minas Gerais, v. 34, n. 1, p. 99-101, 2001.

QUADROS, R. M.; MARQUES, S.; ARRUDA, A. A. R.; DELFES, P. S. W. R.; MEDEIROS, Í. A. A. Parasitas intestinais em centros de educação infantil municipal de Lages, SC, Brasil. Revista da Sociedade Brasileira de Medicina Tropical, Minas Gerais, v. 37, n. 5, p. 422-423, 2004.

ROCHA, R. S.; SILVA, J. G.; PEIXOTO, S. V.; CALDEIRA, R. L.; FIRMO, J. O. A.; CARVALHO, O. S.; KATZ, N. Avaliação da esquistossomose e de outras parasitoses intestinais, em escolares do município de Bambuí, Minas Gerais, Brasil. Revista da Sociedade Brasileira de Medicina Tropical, Uberaba, v. 33, v. 5, p. 431-436, 2000.

ROCHA, T. J. M.; BRAZ, J. C.; CALHEIROS, C. M. L. Parasitismo intestinal em uma comunidade carente do município de Barra de Santo Antônio, estado de Alagoas. Revista Eletrônica de Farmácia, Goiânia, v. 7, n. 3, p. 28-33, 2010.

SANTOS, A. S.; MERLINI, L. S. Prevalência de enteroparasitoses na população do município de Maria Helena, Paraná. Ciências \& Saúde Coletiva, Rio de Janeiro, v. 5, n. 3, p. 899-905, 2010.

VIDAL, A. M. B.; CATAPANI, W. R. Enzymelinked immunosorbent assay immunoassaying versus microscopy: advantages and drawbacks for diagnosing giardiasis. São Paulo Medical Journal, São Paulo, v. 123, n. 6, p. 282-285, 2005.

ZAIDEN, M. F.; SANTOS, B. M. O.; CANO, M. A. T.; NASCIF JÚNIOR, I. A. Epidemiologia das parasitoses intestinais em crianças de creches de Rio Verde-GO. Medicina, Ribeirão Preto, v. 41, n. 2, p. 182-187, 2008.
ZOCHIO, L. B.; CHAVES, P. C.; MONTEIRO, C. P.; FONTOLAN, O. L.; DALPINO, D. Prevalência de parasitas intestinais em crianças do Centro de Convivência Infantil e da EMEI Venâncio Ramalho Guedes de Azevedo do Instituto "Lauro de Souza Lima" (ILSL) Bauru/SP. NewsLab, São Paulo, ed. 79, p. 114-120, 2006.
Recebido em: 20 jul. 2015. Aceito em: 19 out. 2015. 
